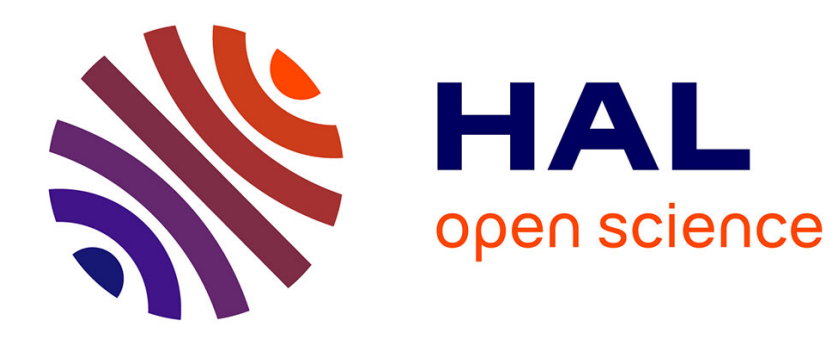

\title{
Hong Kong, une voix démocratique en Chine
}

Philippe Le Corre

\section{To cite this version:}

Philippe Le Corre. Hong Kong, une voix démocratique en Chine. 2020. hal-02476818

\section{HAL Id: hal-02476818 \\ https://hal.science/hal-02476818}

Submitted on 13 Feb 2020

HAL is a multi-disciplinary open access archive for the deposit and dissemination of scientific research documents, whether they are published or not. The documents may come from teaching and research institutions in France or abroad, or from public or private research centers.
L'archive ouverte pluridisciplinaire HAL, est destinée au dépôt et à la diffusion de documents scientifiques de niveau recherche, publiés ou non, émanant des établissements d'enseignement et de recherche français ou étrangers, des laboratoires publics ou privés. 


\section{HONG KONG, UNE VOIX DÉMOCRATIQUE EN CHINE}

Philippe LE CORRE

Hong Kong connait depuis plusieurs mois des manifestations d'hostilité à l'égard du gouvernement chinois. La poussée démocratique ne faiblit pas et pourrait avoir des conséquences politiques lors des élections à venir cette année. Pour comprendre l'évolution de la situation, il faut prendre aussi en compte la recomposition économique de l'ensemble de la région, où Hong Kong n'est plus nécessairement en pointe.

$\mathrm{H}$ ong Kong est unique. Même si la formule magique de l'écrivain Han Suyin " a borrowed place, on borrowed time $»^{1}$ paraît désuète depuis la rétrocession du territoire à la Chine, le ler juillet 1997, l'ancienne colonie britannique semble perpétuellement suspendue à un calendrier incertain ${ }^{2}$. Surtout, malgré de longs mois de crise politico-sociale et de violences, Hong Kong a montré qu'elle n'avait rien perdu de sa différence. Même dans la tourmente, face à un régime chinois déterminé à bloquer toute velléité démocratique, sa population sait attirer l'attention internationale et médiatique, ce qui la distingue de n'importe quel autre territoire chinois - y compris ceux qui jouissent d'un statut particulier: du Tibet à la Mongolie intérieure, en passant par Macao et surtout le Xinjiang, où près d'un million de ressortissants de la minorité ouïgoure sont enfermés dans des camps de rééducation. Aucune région n'a su capter l'attention de la sorte.

1. "Un lieu d'emprunt, vivant au jour le jour. " L'expression anglaise avait été reprise par Richard Hughes, un journaliste basé à Hong Kong.

2. Cf. Ph. Le Corre, Après Hong Kong, chinois et cosmopolite, un pays deux systèmes?, Autrement, 1997. 


\section{Un territoire agité}

Depuis le printemps 2019, Hong Kong fait à nouveau la Une des médias. Elle occupe la scène au Congrès américain, à la Chambre des Communes britannique, au Parlement européen à Strasbourg. Elle agite les think tanks, inquiète aussi le secteur de la finance. Le Parti communiste chinois (PCC) et Xi Jinping, son tout-puissant secrétaire général depuis 2013, se seraient bien passés de cette publicité: plus de 1000 manifestations, 6200 arrestations (dont un tiers sur des individus de moins de 18 ans), de nombreux blessés, des bâtiments gouvernementaux endommagés et l'image de la place financière de Hong Kong - prétendument peu portée sur la politique, mais fièrement revendicatrice de ses droits - durablement changée par un mouvement diffus, doté d'une tactique originale symbolisée par l'expression du kung-fu « être de l'eau $»^{3}$, ce qui permet de multiplier les foyers de protestation, et d'une philosophie clairement ancrée dans la défense du haut degré d'autonomie du territoire.

Au départ, un fait divers: l'assassinat d'une jeune citoyenne de Hong Kong par son compagnon lors d'un voyage à Taïwan, en 2018. Le meurtrier rentre à Hong Kong, se rend aux autorités, puis passe onze mois en prison. Mais il ne peut être extradé pour être jugé à Taipei, en l'absence d'un traité d'extradition entre Hong Kong, région administrative spéciale (RAS) chinoise, et l'île de Taïwan, autonome depuis 1949. C'est à partir de là que Carrie Lam, cheffe de l'exécutif local choisie par Pékin, déclare avoir lancé son projet de loi sur l'extradition. C'est ce texte, amendement à la législation de Hong Kong, qui déclenche les foudres de la population. Les Hongkongais soupçonnent Carrie Lam et son administration de vouloir en fait mettre en place une procédure d'extradition vers la Chine continentale, ce qui constituerait une rupture très claire du système judiciaire " autonome " dont est censé jouir Hong Kong jusqu'en 2047, conformément à la signature de la déclaration sino-britannique de 1984, et de la "Mini-constitution» (Loi fondamentale) qui en est issue. Le nouveau statut doit durer en principe cinquante ans.

En réalité, comme l'ont montré plusieurs enquêtes précises ${ }^{4}$, c'est

3. Formule attribuée à Bruce Lee, star incontestée du kung-fu: "Vide ton esprit, soie comme de l'eau, mon ami. "

4. Enquête "How murder, kidnappings and miscalculation set off Hong Kong's revolt ", Reuters, 20 décembre 2019. 
bien le gouvernement central qui avait incité Hong Kong à mettre en place la législation sur l'extradition, car elle participait d'un renforcement de l'emprise chinoise sur le territoire, souhaité par Pékin depuis de nombreuses années. En 2003 déjà, les autorités avaient cherché à mettre en ouvre l'article 23 de la Loi fondamentale qui aurait permis d'envoyer toute personne soupçonnée de crime se faire juger devant un tribunal chinois. À l'époque, le gouvernement de la RAS avait dû renoncer.

Mais c'était avant Xi Jinping. Depuis 2012, les autorités centrales sont devenues moins tolérantes vis-à-vis des critiques, à l'instar de la vague de répression qui sévit à l'encontre des milieux intellectuels ou dissidents dans le reste du pays. En 2015, la disparition de cinq libraires de Hong Kong ${ }^{5}$, kidnappés et envoyés en Chine continentale, avait défrayé la chronique. À la suite du tollé médiatique soulevé ensuite par le rapt mystérieux en plein centre de Hong Kong d'un homme d'affaires sino-canadien en 2017, Xiao Jianhua, de hauts responsables du Parti communiste chinois commencèrent à faire pression sur la RAS pour qu'elle mette en place un système d'extradition avec la Chine.

Deux ans plus tard, alors que le gouvernement s'apprêtait à faire passer le texte en force devant le Conseil législatif, les Hongkongais n’ont pas tardé à réagir, manifestant bruyamment leur opposition. Dès le mois de juin 2019, période où traditionnellement des habitants du territoire commémorent les sanglants événements de Tian'anmen ${ }^{6}$, les manifestations ont pris un tour beaucoup plus local: le 9 juin, plus d'un million de personnes marchent pour exiger le retrait du texte, et près de deux millions le 16 juin. Face au refus du gouvernement, les manifestations pacifiques se sont multipliées pendant l'été 2019 puis ont pris la forme d'actions plus violentes contre les bâtiments gouvernementaux et contre des institutions chinoises (boutiques, agences de voyages de Chine continentale).

Le $1^{\text {er }}$ juillet, un groupe pénètre dans les locaux du Conseil législatif et les symboles de l'État chinois sont conspués. Le 21 juillet, des manifestants sont attaqués par des hommes en blanc dont on peut

5. Cette affaire est communément appelée "l'affaire des disparitions de Causeway Bay ", en référence à l'adresse de la librairie dissidente, spécialisée dans les ouvrages sur la vie privée des hauts dirigeants chinois.

6. Dans la nuit du 3 au 4 juin 1989, plus d'un millier de personnes sont tuées aux abords de la place Tian'anmen à Pékin, après de longues semaines de manifestations antigouvernementales dans la capitale et ailleurs. Hong Kong avait dès le début apporté son soutien au mouvement prodémocratique. 
penser qu'ils appartiennent au crime organisé. La police laisse faire. L'aéroport international est ensuite occupé, par deux fois. Durant l'automne 2019, les heurts se multiplient, notamment aux abords des campus, ceci malgré le retrait définitif du projet par Carrie Lam. L'affrontement est violent entre des centaines de milliers de manifestants, souvent très jeunes (mais soutenus par leurs aînés), et une police très équipée ayant reçu des renforts de l'autre côté de la frontière, et particulièrement brutale. Rarement la jeunesse hongkongaise n'a été aussi déterminée, prête à en découdre malgré les risques certains et les arrestations multiples 7 . En outre, les menaces contre ceux qui sympathisent avec le mouvement, y compris des adolescents, pleuvent. Ce sont les unités de police mobile qui sont chargées de restaurer l'ordre, en dépit des spéculations laissant entrevoir une intervention armée, plus de trente ans après le massacre de Tian'anmen à Pékin. Les dirigeants du Parti communiste chinois, suffisamment jeunes pour se souvenir du boycott dont la Chine fut l'objet en 1989, optent pour une approche plus insidieuse, sur la durée.

\section{La stratégie de Pékin}

Politiquement, la stratégie du gouvernement de Pékin ${ }^{8}$ aura été principalement de soutenir l'administration locale (la mettre en cause serait se dédire, ce qui est impossible pour le Parti communiste). On qualifie les manifestants de "terroristes » et d'« émeutiers » et on les accuse de collusion avec des « forces étrangères » (black hands) qui les financeraient... ce qui n’a jamais été prouvé. Les États-Unis sont dans le collimateur. À plusieurs reprises, ils ont reçu des porte-parole de Hong Kong tels que l'avocat Martin Lee, figure historique du mouvement démocratique depuis les années 1980, le syndicaliste Lee Cheukyan ou le jeune Nathan Law, 26 ans, ancien du mouvement des "parapluies » de 2014 et dont le siège de député gagné de haute lutte au suffrage universel lui fut retiré en $2016^{9}$. Surtout, le président

7. Alec Ash, "Hong Kong's tears of anger: How a democracy movement radicalized ", Los Angeles Review of Books, 20 décembre 2019, consultable sur https://lareviewofbooks.org/

8. Cf. "Xi Focus: Xi meets HKSAR chief executive ", Xinhuanet, 5 novembre 2019, consultable sur www.xinhuanet.com/

9. En 2016, six députés du camp démocrate se voient destitués par le gouvernement de Hong Kong, principalement pour avoir " manqué de respect " au régime chinois. 
Donald Trump a ratifié, le 27 novembre $2019^{10}$, le Hong Kong Human Rights and Democracy Act of 2019 (HKHRDA), une loi votée unanimement par le Congrès et ayant le pouvoir de sanctionner commercialement les autorités de Hong Kong en cas de violations des droits de l'homme. Cette décision a provoqué l'ire de Pékin, qui a accusé Washington de "soutenir des criminels ». Pourtant, cela n'a pas empêché les négociations commerciales sino-américaines de se poursuivre...

L'économie est le nerf de la guerre pour Hong Kong. Pendant des années, les entreprises du territoire ont investi en Chine. En 1997, $70 \%$ des investissements " étrangers » étaient effectués par des entreprises de l'ancienne colonie qui offrait également à la Chine $70 \%$ de ses revenus touristiques. Outre les "tycoons ", présents au Guangdong dès les années 1980, plus de 4000 entreprises de Hong Kong avaient délocalisé, employant plus de cinq millions d'ouvriers chinois ${ }^{11}$. De nombreuses villes du delta de la rivière des Perles servirent de bases arrière manufacturières aux entreprises de Hong Kong, qui ne s'en plaignirent pas.

Or, depuis quelques années, c'est Hong Kong qui ressemble à la Chine du Sud et non l'inverse ${ }^{12}$. Face aux 60 millions d'habitants de cette région, que pèsent les 7,5 millions de Hongkongais, dont un million sont en fait des « immigrés » arrivés depuis 1997 après avoir traversé la frontière légalement, sous les encouragements de la Chine communiste? Économiquement, Hong Kong est devenue presque exclusivement un centre de services, toujours dynamique, mais concurrencé par Shenzhen, l'ancienne zone économique spéciale créée de toutes pièces par Pékin de l'autre côté de la frontière en 1979, et dont la population est passée depuis, de 50000 à 15 millions d'habitants. Shenzhen héberge en outre de grandes sociétés chinoises telles que ZTE, Huawei, Tencent et China Merchants Group, et compterait autant de sièges d'entreprises du classement Fortune 500 (multinationales comprises) que Hong Kong elle-même! Dans une étude menée par PricewaterhouseCoopers $(\mathrm{PwC})$ et China Development Research Foundation (CDRF), "Chinese Cities of Opportunity

10. Emily Cochrane, "Trump signs Hong Kong Democracy Legislation that has angered China ", The New York Times, 27 novembre 2019.

11. Ph. Le Corre, "Hong Kong, rétrocession à la Chine ", Études, n 3866, juin 1997, p. 728.

12. Ph. Le Corre, "Hong Kong noyée dans la rivière des Perles, vingt ans après la rétrocession ", Asialyst, 29 juin 2017, consultable sur https://asialyst.com/fr/ 
$2017 »^{13}$, Canton et Shenzhen s'adjugeaient les premières places du classement général et en particulier dans huit des dix catégories de l'étude, dont la facilité à faire des affaires, le capital intellectuel et l'innovation, la technologie et le poids économique. Hong Kong ne faisait pas partie de l'étude.

\section{Vit-on mieux à Hong Kong?}

C'est l'un des maillons faibles de Pékin dans sa communication hasardeuse avec les habitants du territoire rentré dans le giron de la mère patrie en 1997. Comment expliquer que l'économie de Hong Kong ne progresse pas, vingt-deux ans après la rétrocession? Seulement 3,8 \% de croissance en 2016, alors que le PNB de la Chine progresse officiellement de $6,5 \%$. En outre, les problèmes sociaux s'accumulent malgré le haut degré d'éducation des Hongkongais, souvent bilingues ou trilingues. Le coefficient de Gini, qui mesure l'inégalité de la répartition des revenus, a atteint un nouveau sommet de 0,539 en 2016, et les prix de l'immobilier - depuis longtemps contrôlé par une dizaine de familles richissimes, ou par des groupes continentaux n'ont jamais baissé. Il est devenu de plus en plus difficile pour une famille de la classe moyenne d'acquérir un appartement: à titre d'exemple, pour un espace de $40 \mathrm{~m} 2$ dans le quartier de Kowloon, le coût moyen est de 4,34 millions de dollars de Hong Kong (502000 euros) alors que le revenu mensuel tourne autour de 2000 euros.

Mais si l'on en croit la presse officielle chinoise, ce serait « la politique locale » qui " empêcherait le développement économique de Hong Kong ", comme semble regretter le China Daily, organe de presse du Parti communiste chinois ${ }^{14}$. Les exigences du clan démocratique auraient prétendument "entravé » la volonté de réforme économique et sociale du gouvernement et l'intégration du territoire dans le projet pharaonique de Xi Jinping: le Belt and Road Initiative ${ }^{15}$ (BRI), dont Hong Kong avait pourtant tenté de devenir une base logistique et

13. PricewaterhouseCoopers et China Development Research Foundation, " Chinese Cities of Opportunities 2017 ", PwC Chine, 17 mars 2017, consultable sur www.pwccn.com/en.html

14. "Behind Hong Kong's chaos lie deep-seated social problems ", China Daily, 8 septembre 2019.

15. En français, la "ceinture et la route ", ou " Nouvelle route de la soie ", mégaprojet d'infrastructures destinées à relier la Chine à l'Europe et aux autres continents. Cf. Jean-Pierre Cabestan, "Les nouvelles routes de la soie ", Études, n4266, décembre 2019, pp. 19-30. 
financière dès 2015. Or, si toutes les grandes institutions du BRI se trouvent basées à Pékin ou à Shanghai ${ }^{16}$, on voit mal comment Hong Kong pourrait trouver sa place. En revanche, un autre mégaprojet, la " grande baie »-comprenant voies ferrées, ponts, ports, aéroports et autoroutes reliant Hong Kong à Canton (capitale de la province de Guangdong), à Macao et à plusieurs villes importantes du Sud concerne directement l'ancienne colonie britannique. L'objectif est tout simplement de diluer Hong Kong dans une immense région et de réaliser l'intégration chère à l'ancien dirigeant chinois Deng Xiaoping (1904-1997), de préférence bien avant la date fatidique de 2047.

$\mathrm{Vu}$ de Pékin, Hong Kong est un chaudron incontrôlable qu'il convient de brider, alors même que les entreprises d'État chinoises (state-owned enterprises of China ou SOE) utilisent, depuis plusieurs décennies, la place financière pour de multiples transactions internationales (fusions acquisitions, introductions en Bourse, levées de fonds). Mais la montée en puissance de Shenzhen et surtout de Shanghai, plus au nord, n'est guère encourageante pour Hong Kong dont on voit mal comment elle resterait la plaque tournante régionale qu'elle a longtemps été. L'ambition de Pékin est de réduire l'importance de Hong Kong, plutôt que d'en préserver les traits spécifiques. À cet égard, la création de nouvelles zones franches illustre la volonté chinoise d'attirer des investissements directs étrangers (IDE) sur tout le continent, et pas seulement dans le Sud. Shanghai, bien sûr, a été la première municipalité à obtenir le droit d'ouvrir sa propre zone franche en 2013, une grosse déception pour Hong Kong. Aujourd'hui, la Chine n'en compte pas moins de onze.

\section{Que va-t-il se passer?}

Dans la tempête, Pékin n'a pas sacrifié Carrie Lam, la très impopulaire cheffe de l'exécutif à l'origine du plus grand mouvement démocratique qu'ait connu Hong Kong depuis les années 1960. Même si le divorce entre son administration et la population est complet, la désavouer eut été une manière inacceptable de perdre la face pour le gouvernement central. Mais il est peu probable qu'elle finisse son mandat en 2022, d'autant que les élections législatives prévues en sep- 
tembre 2020 s'annoncent difficiles.

Malgré le retrait formel du texte sur l'extradition, les manifestants n'ont jamais cessé leurs actions en 2019, y compris pendant la Fête nationale chinoise, le ler octobre. D'une demande unique - le retrait de la loi - les manifestants exigent désormais « cinq demandes, pas une de moins »: retrait de l'article sur l'extradition, seule demande exaucée; mise en place d'une commission sur les violences policières; fin du qualificatif " d'émeutier »; amnistie des personnes arrêtées et suffrage universel pour l'élection du chef de l'exécutif et du Conseil législatif (Legislative Council ou LegCo).

Depuis 1997, Carrie Lam et ses trois prédécesseurs ont été désignés par un comité de 1200 personnes majoritairement choisies par Pékin, cependant que le LegCo se compose actuellement de la manière suivante: trente membres désignés par des circonscriptions professionnelles et quarante élus au suffrage universel (les «pro-Pékin » détenant une majorité de sièges depuis 2016). Or les accords sino-britanniques de 1997 prévoyaient une élection démocratique complète de ces instances au suffrage universel direct, ce que les autorités chinoises ont toujours refusé. En 2014, la Chine avait tenté - sans succès - de proposer l'élection d'un chef de l'exécutif parmi trois candidats triés sur le volet par un comité progouvernemental. Cette offre de la dernière chance avait mené au mouvement des parapluies d'octobre, exigeant de véritables élections... ce qui n'avait guère de chances d'aboutir.

Toutefois, un épisode récent a stupéfié Pékin: les élections locales de Hong Kong, le 24 novembre 2019, ont permis aux démocrates de remporter 17 conseils de district sur 18 , avec un taux de participation record de $71 \%$. Les partis pro-Pékin, à commencer par le Democratic Alliance for the Betterment and Progress of Hong Kong (DAB), ont subi un échec cuisant. Or ces conseils de district d'ordinaire peu stratégiques ont depuis peu le pouvoir de désigner cinq membres du LegCo, ce qui pourrait avoir des conséquences importantes lors des prochaines élections législatives, en septembre 2020.

L'année qui commence devrait voir se poursuivre le mouvement démocratique, sans doute stimulé par l'impact international qu'il a soulevé. Néanmoins, la société hongkongaise est aussi profondément traumatisée par les violences de l'année 2019. Du côté de l'Église catholique, les évêques n'ont pas hésité à défier Pékin, qui ne reconnaît pas le Vatican, même si ce dernier, depuis l'arrivée du pape François, 
a fait plusieurs pas en direction de la Chine. Le cardinal Joseph Zen Ze-kiun, ancien archevêque de Hong Kong qui a toujours pris la défense des catholiques en Chine, dont beaucoup doivent se réunir clandestinement, n’a pas hésité à critiquer la position de Rome. Évêque auxiliaire de Hong Kong, Joseph Ha Chi-shing a carrément pris parti pour les manifestants, sans toutefois recevoir l'appui de Rome qui semble vouloir éviter toute controverse. Là aussi, la situation de Hong Kong, qui compte environ 400000 catholiques (dont Carrie Lam ellemême), pourrait servir de test. De même, à Taïwan, l'île que Pékin cherche à attirer dans son giron, la population reste opposée à tout rapprochement qui la mettrait sous le joug du Parti communiste chinois.

Il est clair que ce dernier est engagé dans une lutte pour sa survie, ce qui inclut la politique répressive des autorités centrales vis-à-vis des régions autonomes et des minorités. Dans le prolongement du discours prononcé par Xi Jinping lors du XIXe congrès du parti en 2017, la mise au pas de ceux qui ne font pas allégeance au pouvoir suprême paraît logique. Mais Hong Kong présente de nombreuses difficultés car le territoire jouit encore d'une certaine autonomie médiatique, judiciaire et administrative qui permet à sa population de faire entendre sa voix. C'est une grande différence par rapport aux Ouïgours ou aux Tibétains, sans parler des citoyens chinois dissidents qui osent défier le régime de l'intérieur.

Après l'élection présidentielle à Taïwan, nul doute que le régime de Pékin va chercher à reprendre la main à Hong Kong en essayant de souder tous les soutiens dont il dispose en vue des élections. Mais, en 2019, les nuages se sont amoncelés sur la Chine de Xi Jinping, dont on pensait qu'aucun événement ne pourrait freiner l'ambition, aussi bien en Chine qu'à l'extérieur. Ses grands projets d'infrastructures ont subi des revers. L'économie domestique voit sa croissance ralentir. Et, si l'on en croit la dernière étude de l'excellent Pew Research Center, la perception de la Chine dans les pays développés accuse une forte baisse, malgré les projets de nouvelle route de la soie. Ainsi, les opinions négatives des Français à l'égard de la Chine sont passées de $54 \%$ à $62 \%$ entre 2017 et 2018. Pour le Royaume-Uni, ancienne puissance coloniale à Hong Kong, les opinions négatives passent de $35 \%$ à $55 \%$. L'Allemagne reste stable à environ $54 \%$ d'opinions négatives de 
la Chine ${ }^{17}$. C'est une preuve, s'il en était besoin, que la montée en puissance de l'ancien Empire du Milieu provoque inquiétude et frayeur du Canada au Japon, en passant par l'Australie et les pays européens. Sans pour autant demander l'impossible pour son avenir (à savoir l'indépendance, que sa population n'a d'ailleurs jamais exigée), Hong Kong a joué le rôle étonnant - comme en 1997 - de détonateur et d'aiguillon dans une région où la démocratie n'a pas, en dehors de l'Inde et d'une poignée de pays d'Asie orientale, le vent en poupe. Même si la place financière ne semble pas avoir subi de secousses trop violentes en 2019, les investisseurs internationaux n'ont de cesse d'observer l'évolution de Hong Kong et de se projeter vers des destinations a priori moins risquées, ce qui n'arrangerait pas non plus les affaires d'une Chine conquérante qui s'efforce d'offrir son modèle politique aux pays qui veulent bien l'entendre.

Philippe LE CORRE

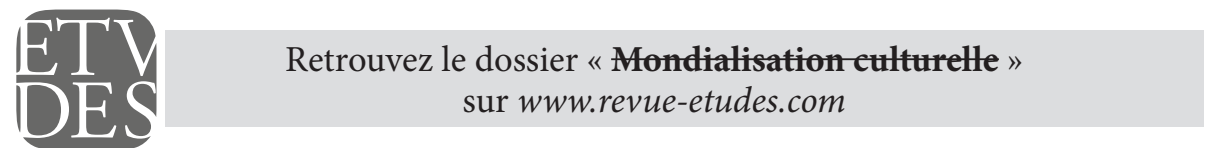

17. Kat Devlin, Christine Huang et Laura Silver, "How people view China " dans " Attitudes toward China ", Pew Research Center, 5 décembre 2019, consultable sur www.pewresearch.org 